\title{
Practical needle selection for Vienna-style applicators: improving therapeutic ratio in hybrid intracavitary-interstitial brachytherapy
}

\author{
David A. Martin, BS, Neil K. Taunk, MD, MSCTS, Shibu Anamalayil, MS, Vatsal Mangal, Jaclyn Marcel, MS, \\ Emily Hubley, MSc \\ Department of Radiation Oncology, Perelman School of Medicine, University of Pennsylvania, Philadelphia, Pennsylvania, USA
}

\begin{abstract}
Purpose: Hybrid intracavitary and interstitial (IC/IS) applicators improve dose distribution compared to traditional IC applicators in cervical high-dose-rate (HDR) brachytherapy. There is a learning curve to these applicators, and initial standard needle insertion patterns have not been well-established. In this study, we quantified dosimetric benefits of IC/IS applicators, and offer practical initial interstitial needle-selection, insertion depths, and dwell position recommendations.

Material and methods: Fifteen patients previously treated with a tandem and ring IC applicator and magnetic resonance (MR)-guidance were re-planned at first fraction using a digital template of Vienna-style interstitial needles. IC/IS plans maintained identical high-risk clinical target volume (HR-CTV) $\mathrm{D}_{90 \%}$ while reducing dose to organs at risk (OARs). To assess the validity of planning using virtual needles, virtual needle templates were overlaid on twelve clinical IC/IS plans, and the displacements between 40 physical and virtual needles were measured at $3 \mathrm{~cm}$ depth.

Results: The median HR-CTV volume in the present study was $19.6 \mathrm{cc}$ (range, 6.6-60.5 cc). HR-CTV $\mathrm{D}_{90 \%}$ was maintained in all re-plans. Median bladder $\mathrm{D}_{2 \mathrm{cc}}$ decreased from 5.4 Gy per fraction to $4.8 \mathrm{~Gy}(p=0.003)$; median rectum $\mathrm{D}_{2 \mathrm{cc}}$ decreased from 2.4 Gy per fraction to $2.0 \mathrm{~Gy}(p=0.007)$. We suggest that a standard loading pattern should include needles in lateral channels 4,5, and 9, 10 inserted $3 \mathrm{~cm}$ deep, with dwell times $<20 \%$ of the combined tandem and ring dwells. The mean displacement between planned and physical needles was $1.8 \mathrm{~mm}$. All needles but three deviated less than $3.3 \mathrm{~mm}$, demonstrating the validity of re-planning with virtual needles.

Conclusions: Hybrid IC/IS applicators maintain excellent $\mathrm{D}_{90 \%}$ coverage while improving dose to OARs compared to IC-only applicators, even in non-bulky HR-CTVs. We offer practical recommendations for needle selection, insertion depth, and relative weighting for Vienna-style applicators in small HR-CTVs. These results support previous publications, offering practical recommendations for users of Vienna-style hybrid applicators.
\end{abstract}

J Contemp Brachytherapy 2021; 13, 5: 533-540 DOI: https://doi.org/10.5114/jcb.2021.110348

Key words: brachytherapy, cervical cancer, image-guided brachytherapy, Vienna applicator, intracavitary, interstitial.

\section{Purpose}

In the treatment of cervical cancer, consensus statements recommend a cumulative dose (external beam + brachytherapy) of 80-90 Gy equivalent dose in two Gray fractions, with $\alpha / \beta$ ratio of 10 (EQD2 10 ), covering $90 \%$ of high-risk clinical target volume (HR-CTV) while limiting $\mathrm{D}_{2 \mathrm{cc}}$ of the rectum, sigmoid, and bladder to $65 \mathrm{~Gy}, 70 \mathrm{~Gy}$, and $80 \mathrm{~Gy}$, respectively $\left(\mathrm{EQD}_{3}\right)[1,2]$. Achieving sufficient target coverage while limiting organ at risk (OAR) doses, especially for asymmetric or large HR-CTVs, is not always achievable with standard tandem and ring or tandem and ovoid intracavitary (IC) brachytherapy applicators [3]. For patients with bulky lesions, where a standard IC applicator would not allow for sufficient target coverage and OAR sparing, an interstitial implant with a perineal template can be used; however, these procedures and the associated patient management are complex [4].

The Vienna-style ring applicator is a hybrid intracavitary/interstitial (IC/IS) applicator for high-dose-rate (HDR) or pulsed-dose-rate (PDR) brachytherapy. It is similar in appearance to a tandem and ring applicator, except the ring cap has holes in which up to nine titanium or plastic interstitial needles can be inserted. The unique construction of Vienna-style applicator classifies it as both an intracavitary and interstitial device [5]. Hybrid IC/IS applicators are most suited for irregular target volumes in some patients [5-7], and both procedure and patient management requirements may be less complex than a template-based interstitial implant.
Address for correspondence: Emily Hubley, MSc, Department of Radiation Oncology, Perelman School of Medicine, University of Pennsylvania, 3400 Civic Center Boulevard, Philadelphia, PA 19104, USA, phone: +1-215-615-6767, $₫$ e-mail: emily.hubley2@pennmedicine.upenn.edu
Received: 23.02 .2021

Accepted: 01.09 .2021 Published: 29.10.2021 
Data from RetroEMBRACE study has demonstrated higher local control for IC/IS applicators compared to IC-only applicators in cases of HR-CTVs larger than $30 \mathrm{~cm}^{3}$ [8]. Dosimetry improvements have been shown in patients with HR-CTVs smaller than $30 \mathrm{~cm}^{3}$ using a hybrid IC/IS applicator compared to a conventional IC applicator [9]. The current EMBRACE II study suggests potentially even higher dose to HR-CTV and decreased limits to OARs, balancing tumor control with toxicity. However, improved target coverage is difficult to achieve without exceeding dose to organs at risk. Therefore, the emerging body of evidence indicates that the therapeutic ratio may be improved using hybrid applicators.

However, there is a potential learning curve utilizing a new applicator and optimal applicator insertion requires additional physics and physician expertise [10]. Relative loading and weighting for dwell times, how many needles to insert into a patient, and how far to insert them have not yet been well-documented in the literature for hybrid applicators. The primary goal of this study was to determine how simulated optimal needle insertion may yield practical dosimetric benefits to maximize the therapeutic ratio if Vienna-style ring applicator is used in a place of standard tandem and ring applicator.

\section{Material and methods}

\section{Patients}

Fifteen consecutive locally advanced cervical cancer patients treated with definitive chemo-radiotherapy, followed by tandem and ring IC brachytherapy were included in this planning study. All patients presented with squamous cell carcinoma of the cervix and received cisplatin-based 45 Gy image-guided intensity-modulated radiation therapy (IG-IMRT). This was followed by HDR IC brachytherapy,

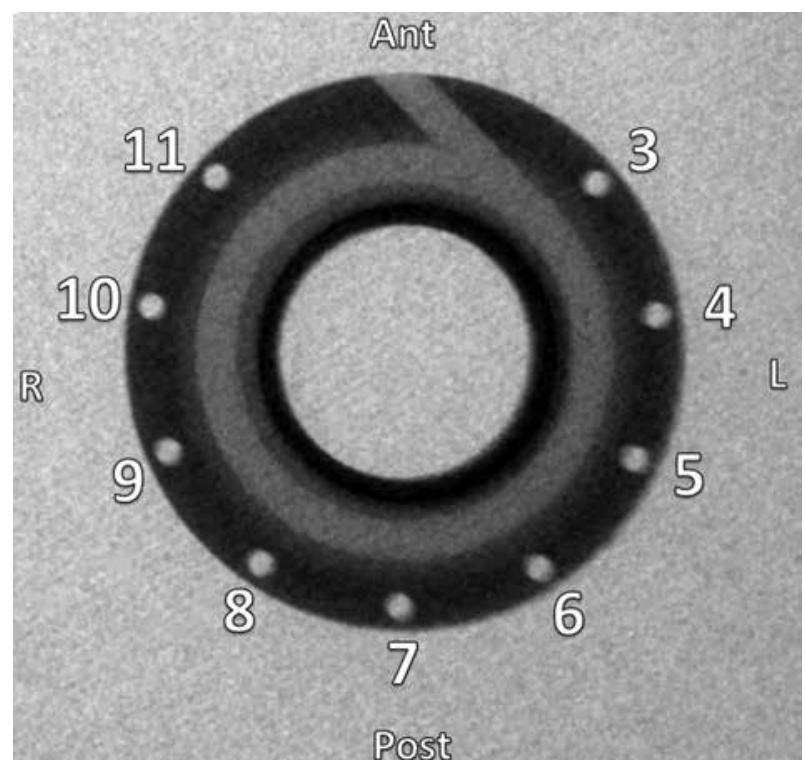

Fig. 1. A planar $\mathrm{kV}$ image of IC/IS ring cap. The plastic cap, through which the needles are inserted fits around the titanium ring applicator. Numbers 3-11 correspond to a needle's channel number and orientation most commonly with 28 Gy in four fractions (12 patients), or less commonly with $27.5 \mathrm{~Gy}$ ( 3 patients) in five fractions, using a titanium tandem and ring applicator with a $5 \mathrm{~mm}$ plastic build-up cap (Mick Radio-Nuclear Instruments, Eckert and Ziegler BEBIG, Berlin, Germany). All patients received CT- and MR-based imaging using a $1.5 \mathrm{~T}$ Espree scanner (Siemens Healthineers AG, Erlangen, Germany). HR-CTV contouring was performed on a T2-weighted oblique MRI series, with slices angled parallel to the ring. MRI was registered to the planning CT using rigid registration, with a focus on the applicator and soft tissue in the immediately adjacent area. Organ at risk (OAR) contouring and applicator reconstruction using solid applicator model placement were done on a $2 \mathrm{~mm}$ slice-thickness CT. Treatment plans were created in Varian BrachyVision version 15.6 (Varian Medical Systems, Palo Alto, CA, USA). For all calculations, an ${ }^{192} \mathrm{Ir}$ source strength of $40700 \mathrm{cGy} \mathrm{cm} / \mathrm{h}$ (10 Ci) was used, and a TG-43 line-source dose calculation formalism was used. CT and calculation voxel sizes were both $12.5 \mathrm{~mm}^{3}(2.5 \mathrm{~mm} \times 2 \mathrm{~mm} \times 2.5 \mathrm{~mm})$. The study was approved by our university's institutional review board.

\section{Vienna-style hybrid applicator}

A virtual implant template was created in BrachyVision, consisting of a maximum of nine needles that may be inserted around the ring in a tandem and ring applicator (Figure 1). Virtual needles consisted of $2 \mathrm{~mm}$ wide and $32 \mathrm{~cm}$ long plastic needles available from Varian, with a dead space of $3.5 \mathrm{~mm}$. The implant template with nine straight needles extending $6 \mathrm{~cm}$ beyond the ring cap was placed around the tandem and ring applicator. The digitized template is shown in Figure 2.

\section{Planning method, re-planning method, and dosimetry criteria}

Patients were retrospectively re-planned on their first fraction treatment plan with virtual IC/IS hybrid implant to demonstrate an initial potential needle and loading pattern for a first fraction IC/IS. To re-plan all of the patients with this new template, a CT image set for each patient's first fraction was loaded, which contained clinical contours for HR-CTV and all OARs, including bladder, rectum, sigmoid, and small bowel. Small bowel was contoured only in patients where the organ was located within $4 \mathrm{~cm}$ of the applicator. The tandem and ring applicator was digitally inserted to match the applicator on CT according to our previously published method, and the Vienna-style template was centered on the ring [11].

Initial treatment plans on the ring and tandem applicator were generated using a standard dwell pattern normalized to point $\mathrm{A}$, using only dwell positions in the tandem and ring applicators $[4,12]$. Then, manual iterative optimization was performed by adding dwell positions in the interstitial needles and on the ring and tandem to achieve the target HR-CTV goal above (minimum 80-85 Gy) and keep $\mathrm{EQD}_{2} \mathrm{D}_{2 \mathrm{cc}}$ doses below the ASTRO Clinical Practice Guidelines ideal limits (rectum, < 65 Gy, bladder, $<80$ Gy, sigmoid, < 70 Gy) [1]. 

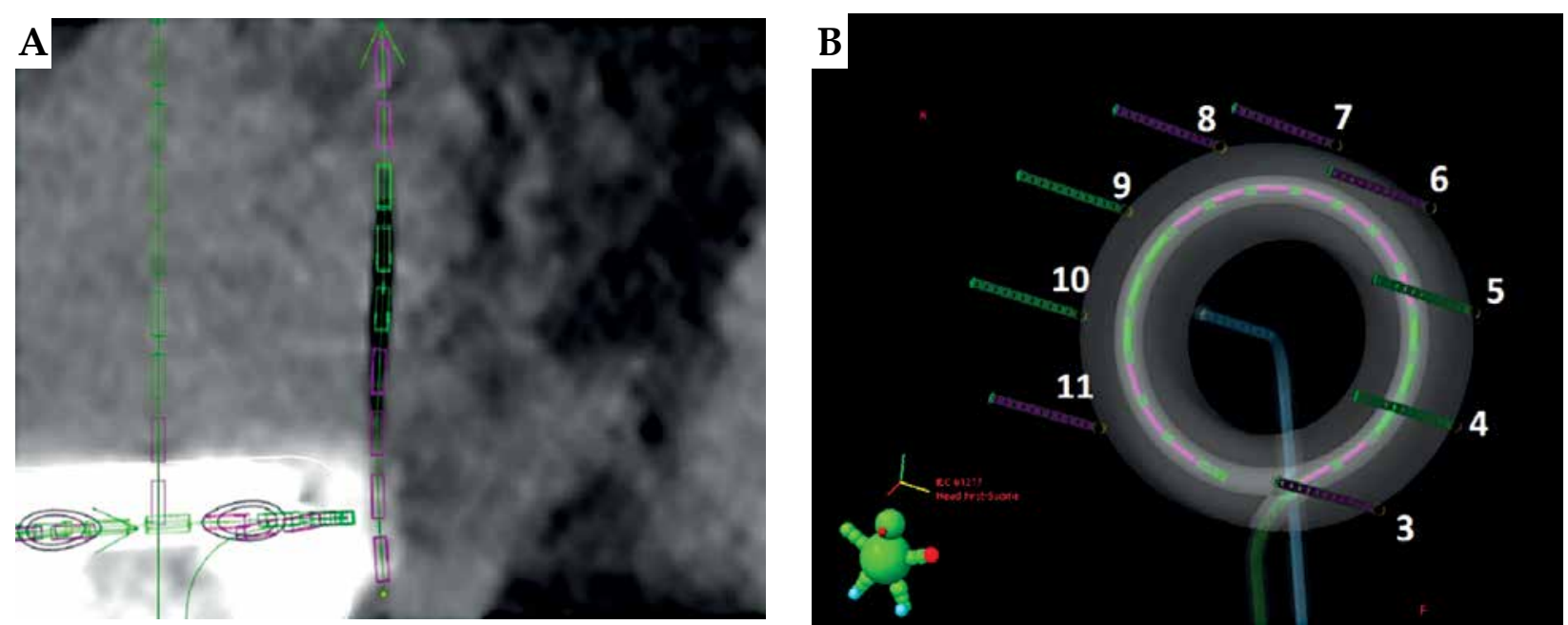

Fig. 2. A Vienna-style needle adjacent to the ring in an example clinical planning CT (A). Notice the slight potential bending of the needle when inserted into a patient. The 3D projection of digital Vienna-style IC/IS applicator with nine straight needles in a tissue-equivalent background (B). The interstitial needle template is centered on the ring.

The doses to the HR-CTV and OARs were reported from the first fraction clinical treatment plan for all IC patients. The goal of this re-planning study was to maintain the same dose to $90 \%$ of HR-CTV ( $\mathrm{D}_{90 \%}$ in Gy) as the clinical plans, while lowering the dose $\left(\mathrm{D}_{2 \mathrm{cc}}\right)$ to OARs, such as bladder, rectum, and sigmoid. Maintaining constant HR-CTV $\mathrm{D}_{90 \%}$ was selected to facilitate a clear comparison between clinical IC plans and re-planned IC/IS plans. In addition, there were no local recurrences reported in this consecutive cohort at a minimum of 2 years follow-up, determined by a 3-month post-treatment positron emission tomography/computed tomography (PET/CT) and serial clinical exam, thus, tumor control dose-response was not explored. Doses to points A and B were reported as well as dose to $100 \%$ of HR-CTV $\left(D_{100 \%}\right)$. Optimizing the plans about $\mathrm{D}_{90 \%}$ was motivated by dose to $\mathrm{D}_{90 \%}$ HR-CTV correlated to local control $[2,3]$.

Once all patients were re-planned and meeting the criteria for HR-CTV, the number of needles need- ed to be inserted in each patient and total dwell time in each needle were recorded. In all re-planned IC/IS plans, the total dwell times in the needles did not exceed $10-20 \%$ of the total dwell time, in accordance with the American Brachytherapy Society consensus recommendations for IC/IS applicators [12]. Doses to all OARs were recorded and compared to clinically used IC plans. EQD2 ${ }_{3}$ (external beam + brachytherapy) $\mathrm{D}_{2 \mathrm{cc}}$ doses were calculated for the bladder, rectum, and sigmoid, based on whether the patient received 4 or 5 fractions of brachytherapy. A two-tailed paired Student's $t$-test was applied comparing IC data to IC/ IS data using Python SciPy Statistical package (version 3.7.4). A $p$-value of $<0.05$ was selected for statistical significance. HR-CTV volume was recorded. Additionally, maximum width in any plane perpendicular to the tandem axis was documented, and HR-CTV maximum length in any plane parallel to the tandem axis were measured and recorded.

Table 1. Comparison of median doses for 15 patients with and without a ring hybrid. A negative difference signifies a decreasing dosimetric parameter of IC/IS plan relative to IC-only plan, and a positive difference indicates an increasing dosimetric parameter of IC/IS plan compared to IC-only plan

DVH criteria Median (range) IC/IS plan doses Median (range) IC plan doses (Gy) Difference (Gy) ( $p$-value)
(Gy)

\begin{tabular}{lccc}
\hline HR-CTV $D_{100 \%}(G y)$ & $5.6(1.9-7.7)$ & $5.6(1.8-7.8)$ & $-0.0(0.256)$ \\
\hline HR-CTV $D_{90 \%}$ (Gy) & $8.1(6.4-11.6)$ & $8.2(6.4-11.6)$ & $-0.1(0.518)$ \\
\hline HR-CTV V ${ }_{100 \%}$ & $99.5 \%(87.1-100 \%)$ & $98.6 \%(88.6-100 \%)$ & $+0.9 \%(0.679)$ \\
\hline PT A left & $6.1(4.3-10.4)$ & $5.6(3.9-7.3)$ & $+0.5(0.033)$ \\
\hline PT A right & $5.7(4.0-6.9)$ & $5.8(3.8-7.4)$ & $-0.1(0.159)$ \\
\hline PT B left & $1.7(1.0-2.4)$ & $1.8(1.0-2.5)$ & $-0.1(0.613)$ \\
\hline PT B right & $1.3(0.7-1.6)$ & $1.6(0.9-1.9)$ & $-0.3(0.004)$ \\
\hline Bladder $D_{2 c c}$ & $4.8(2.5-6.2)$ & $5.4(2.5-6.5)$ & $-0.6(0.003)$ \\
\hline Rectum $D_{2 c c}$ & $2.0(1.2-3.2)$ & $2.4(1.4-5.5)$ & $-0.4(0.007)$ \\
\hline Sigmoid $D_{2 c c}$ & $3.3(1.7-4.9)$ & $3.5(1.7-5.3)$ & $-0.2(0.011)$
\end{tabular}


A

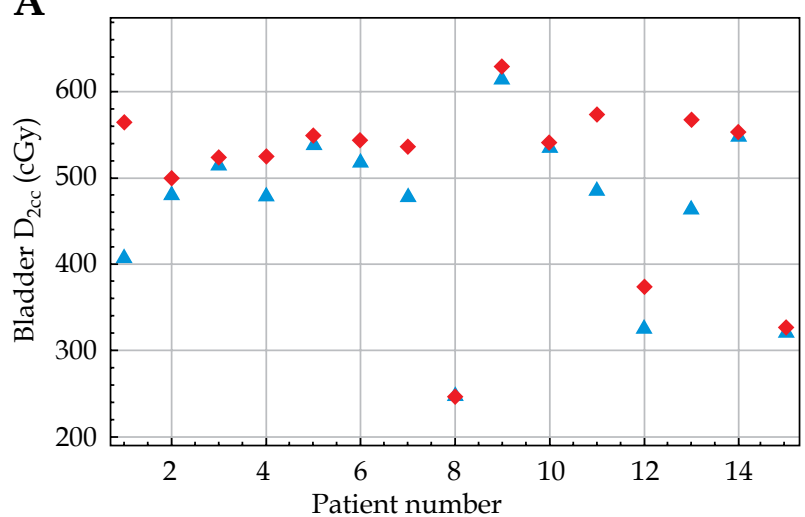

$\Delta$ With IS needles $\bullet$ Without IS needles

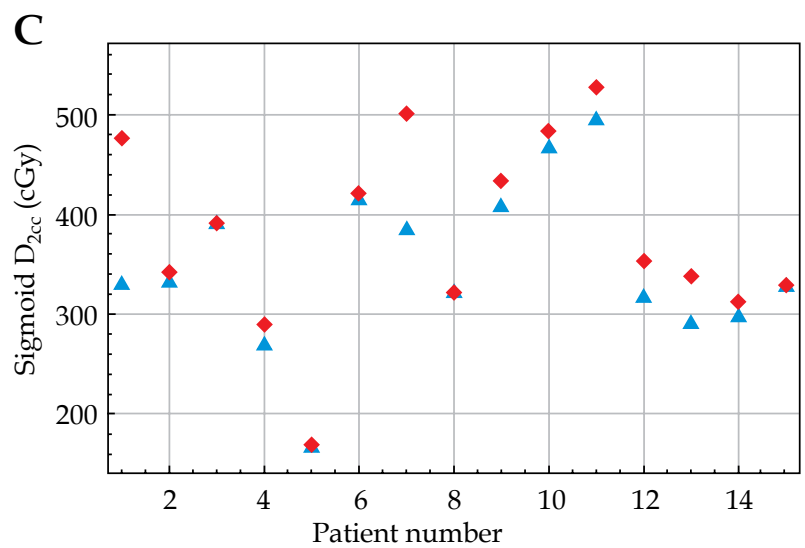

$\Delta$ With IS needles

- Without IS needles

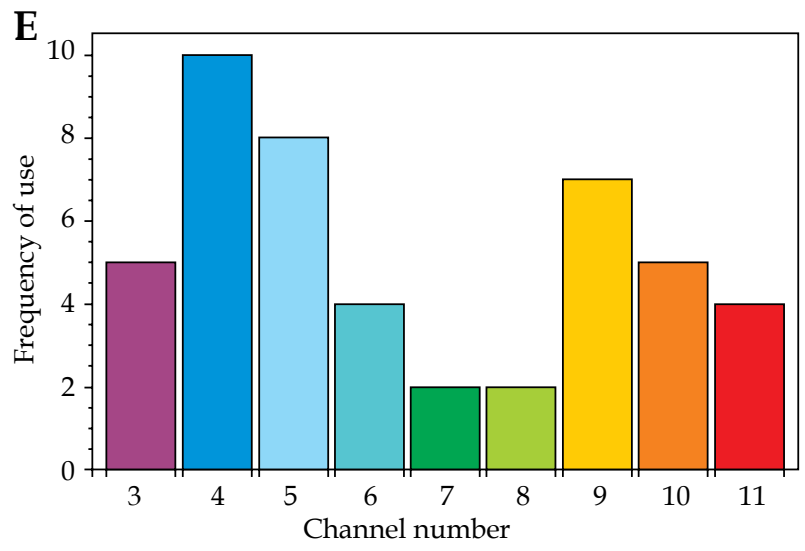

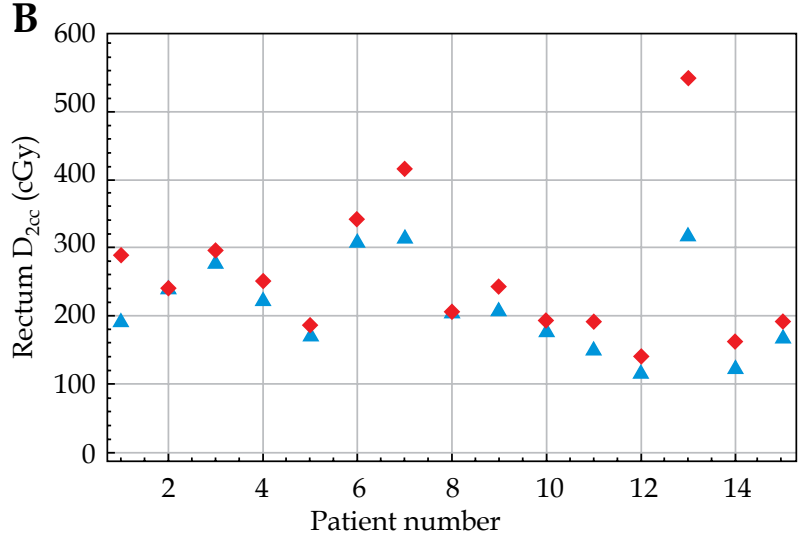

$\Delta$ With IS needles

Without IS needles

D

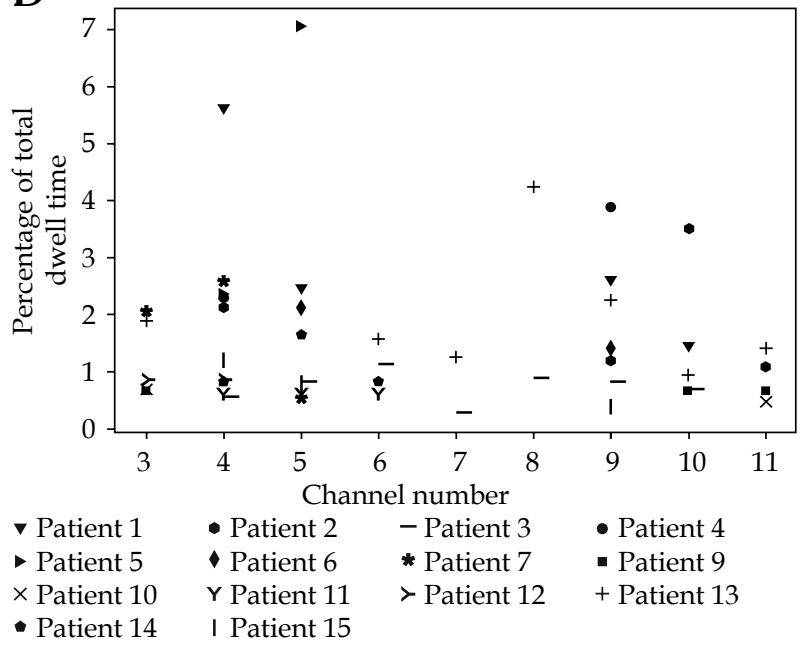

F

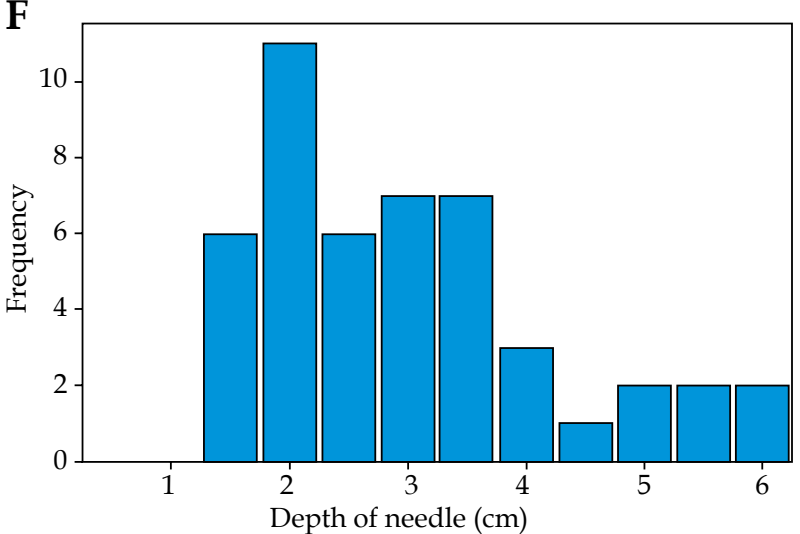

Fig. 3. OAR dose improvement (A-C), total dwell time in each needle per 15 patients (D), how often each channel was loaded (E), depth of needle insertion $(\mathbf{F})$

\section{Evaluating virtual implant template accuracy}

There is natural needle deviation in clinical IC/IS treatment when needles are inserted into tissue (Figure 2A), indicating a need to verify the accuracy of digital re-planning. Here, IC/IS plans were created by placing a virtual implant template containing straight needles, rather than digitizing needles physically placed in the patients. To assess the accuracy of this technique, treatment plans of a different cohort of twelve consecutive patients who had clinical IC/IS plans were used. The virtual implant template was placed, and displacement between the actual needles and virtual needles was measured at $3 \mathrm{~cm}$ past the ring, along the tandem axis. A total of 40 needles in twelve plans were assessed.

\section{Results}

The digital re-planning technique was validated using the digitally inserted needle template compared to 

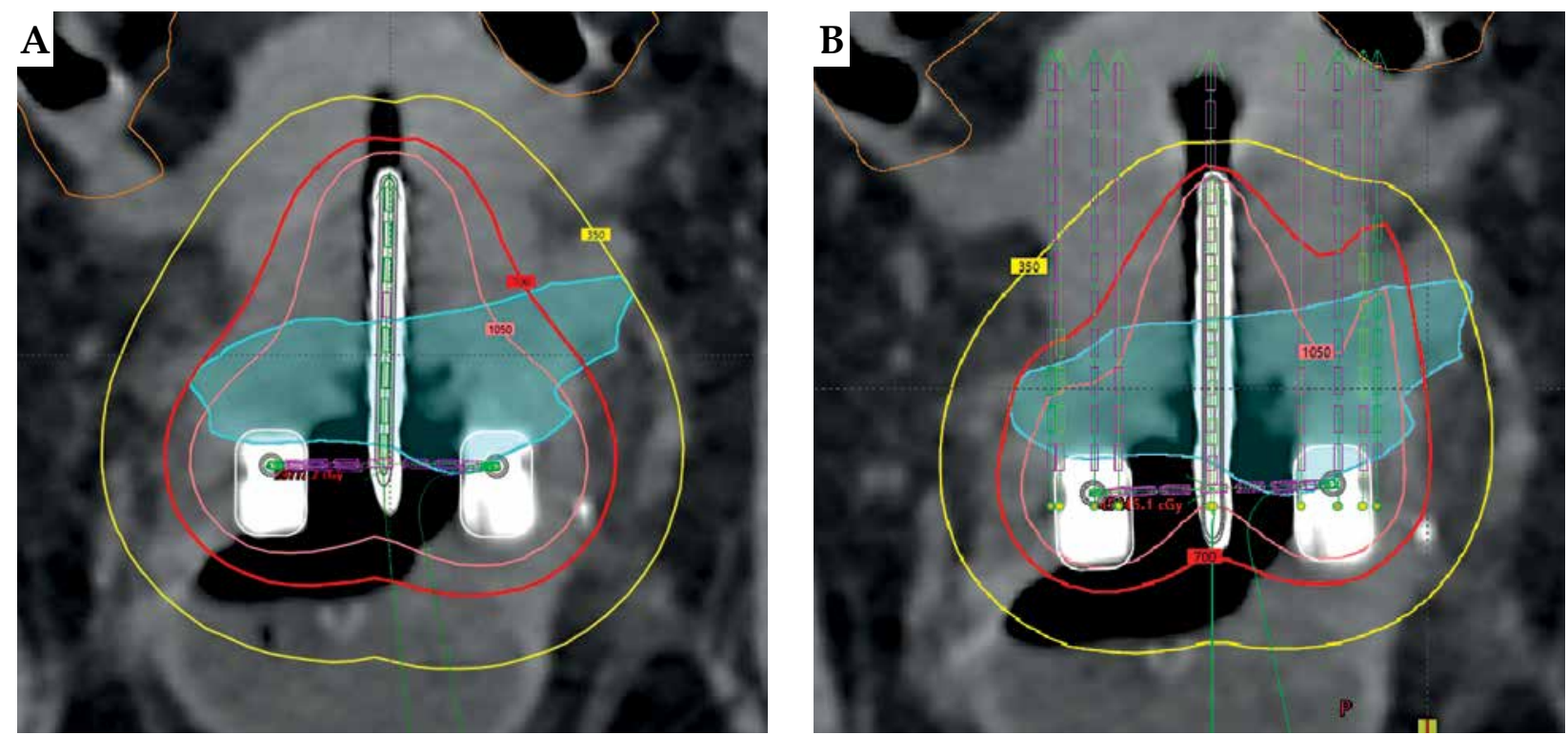

Fig. 4. CT image slices of dose distribution are shown for a tandem and ring clinical plan (A) vs. dose distribution for the same patient but with a ring hybrid used (B). 100\% isodose line (red), 150\% isodose (pink), and 50\% isodose line (yellow)

clinical needles for 40 needles in twelve previously treated IC/IS patients. The template interstitial needles were placed alongside clinical IC/IS treated patients' needles with Vienna hybrid applicator. The average spatial deviation in a needle position at $3 \mathrm{~cm}$ depth was found to be $1.8 \mathrm{~mm}$ in clinical practice (range, 0.0-6.7 $\mathrm{mm}$; standard deviation, $1.4 \mathrm{~mm}$ ). All but three needles deviated less than $3.3 \mathrm{~mm}$. A needle with the largest deviation $(6.7 \mathrm{~mm})$ was outside the target and was not loaded for clinical use. This $1.8 \mathrm{~mm}$ difference between the planned and placed needles was small and comparable to that reported in the literature [13].

The median HR-CTV volume of the 15 digitally re-planned patients in this study was $19.58 \mathrm{cc}$ (range, 6.6-60.5 cc). All patients but one (patient No. 8) in the study exhibited lowered OAR doses in IC/IS plans compared to their former IC clinical plans (Table 1, Figure $3 \mathrm{~A}-\mathrm{C}$ ). Patient No. 8, who received no benefit from the addition of interstitial needles had an HR-CTV volume of $6.6 \mathrm{cc}$. In several patients with asymmetric HR-CTVs, benefits were more obvious (Figure 4). IC/IS applicators proved more versatile to cover HR-CTVs, which extended laterally, yielding an opportunity to scale down or remove some of the upper tandem dwells and ring dwells. Removing dwells in the upper portion of the tandem lowered the dose to the sigmoid and small bowel, while scaling down dwells in the ring lowered rectum and bladder doses. Very small, narrow, or symmetric HR-CTVs did not receive much benefit from the addition of a ring hybrid.

As seen in Table 1, OAR $\mathrm{D}_{2 \mathrm{cc}}$ doses decreased when including the ring hybrid, by around 0.20-0.60 Gy in a single fraction. These first-fraction doses extrapolated to 4 or 5 fraction courses of 7 Gy $\times 4$ or 5.5 Gy $\times 5$ brachytherapy, with 45 Gy EBRT, corresponds to a decrease in mean $\mathrm{EQD}_{2}$ cumulative dose from $77.8 \mathrm{~Gy}$ to $73.4 \mathrm{~Gy} \mathrm{D}_{2 \mathrm{cc}}$ for the bladder, from $56.0 \mathrm{~Gy}$ to $52.4 \mathrm{~Gy} \mathrm{D}_{2 \mathrm{cc}}$ for the rectum, and from $65.4 \mathrm{~Gy}$ to $62.5 \mathrm{~Gy} \mathrm{D}_{2 \mathrm{cc}}$ for the sigmoid.
The median decreases in $\mathrm{D}_{2 \mathrm{cc}}$ cumulative $\mathrm{EQD}_{2}$ were from 79.6 Gy to 73.2 Gy for the bladder, from 53.5 Gy to $51.4 \mathrm{~Gy}$ for the rectum, and from $64.9 \mathrm{~Gy}$ to $62.8 \mathrm{~Gy}$ for the sigmoid. Some patients, like the patient shown in Figure 4, exhibited OARs dose reduction of up to 1 Gy. Another patient (patient No. 8) had an HR-CTV of $6.6 \mathrm{cc}$ and did not benefit from the use of ring hybrid. The reduction of dose to all OARs (rectum, bladder, and sigmoid) was statistically significant, and demonstrated consistent reduction in OAR dose.

The most frequently loaded channels in the replanned IC/IS plans were needles in the lateral positions of channels 4, 5, and 9 (Figure 3E). Other channels were loaded rarely, as placing a needle there would most likely pierce an OAR (e.g., anterior needles potentially piercing an eccentric bladder) or did not provide marked dosimetric benefit. On average, each patient had about 3 needles loaded in the re-planned cases. This was not always the case, as some patients benefitted from needle insertion into almost all of the channels. The average depth of insertion for clinical needles was $3 \mathrm{~cm}$, while the average depth of the highest dwell position in each needle was $2.5 \mathrm{~cm}$. The minimum needle insertion depth was $1.5 \mathrm{~cm}$, as dwell positions within $1 \mathrm{~cm}$ of the ring did not provide dosimetric benefit in any of the plans. The percentage of total dwell time in the needles (Figure 4D) favored the more commonly inserted lateral needles in channel positions 4, 5, 9, and 10. Some of the highest dwell times were found in these more common channels' positions. The percentage of total dwell times in the interstitial needles for all the 15 re-plans was $0-14 \%$, which aligns with consensus recommendation [12].

\section{Discussion}

In the current study, we presented practical initial needle selection for the Vienna-style IC/IS applicator and 
Table 2. HR-CTV geometry, needles loaded, and OAR $D_{2 c c}$ dose decrease for each patient. * indicates 5 fractions of brachytherapy, which was accounted for in total brachytherapy + external beam $\mathrm{EQD}_{2}$ dose calculation. All other patients received 4 fractions of brachytherapy

\begin{tabular}{|c|c|c|c|c|c|c|c|c|c|c|c|}
\hline \multirow[t]{2}{*}{ Patient } & \multirow{2}{*}{$\begin{array}{l}\text { HR-CTV } \\
\text { volume } \\
\text { (cc) }\end{array}$} & \multirow{2}{*}{$\begin{array}{l}\text { HR-CTV } \\
\text { max } \\
\text { width } \\
\text { (cm) }\end{array}$} & \multirow{2}{*}{$\begin{array}{l}\text { HR-CTV } \\
\text { max } \\
\text { height } \\
(\mathrm{cm})\end{array}$} & \multirow{2}{*}{$\begin{array}{c}\text { No. of } \\
\text { needles } \\
\text { loaded }\end{array}$} & \multirow{2}{*}{$\begin{array}{l}\text { Percent- } \\
\text { age of } \\
\text { total } \\
\text { dwell } \\
\text { time in } \\
\text { needles }\end{array}$} & \multicolumn{3}{|c|}{$\begin{array}{c}\text { Decrease in } D_{2 c c} \text { per HDR } \\
\text { fx. (cGy) }\end{array}$} & \multicolumn{3}{|c|}{$\begin{array}{c}\text { Total BT + EBRT EQD } \\
\text { decrease (Gy) }\end{array}$} \\
\hline & & & & & & Bladder & Rectum & Sigmoid & Bladder & Rectum & Sigmoid \\
\hline 1 & 19.05 & 5.8 & 2.7 & $\begin{array}{c}4,5,9 \\
10\end{array}$ & 12.4 & 156.9 & 98.4 & 146.2 & 16.0 & 6.1 & 13.0 \\
\hline 2 & 17.13 & 4.5 & 3.9 & $4,9,10$ & 7.5 & 18.9 & 0.5 & 10.8 & 1.9 & 0.0 & 0.8 \\
\hline 3 & 26.78 & 4.2 & 2.5 & $\begin{array}{c}4,5,6,7 \\
8,9,10\end{array}$ & 5.2 & 9.1 & 20.3 & 1.2 & 1.0 & 1.4 & 0.1 \\
\hline 4 & 13.02 & 3.9 & 2.6 & 4,9 & 6.2 & 46.0 & 29.5 & 21.5 & 4.8 & 1.8 & 1.5 \\
\hline 5 & 8.06 & 3.4 & 2.8 & 4,5 & 9.4 & 10.3 & 16.9 & 3.0 & 1.1 & 0.9 & 0.2 \\
\hline 6 & 12.97 & 2.7 & 3.6 & 5,9 & 3.5 & 26.3 & 34.8 & 7.2 & 2.9 & 2.6 & 0.7 \\
\hline 7 & 20.78 & 4.4 & 2.9 & $3,4,5$ & 5.2 & 58.8 & 103.6 & 117.0 & 6.2 & 8.5 & 11.1 \\
\hline 8 & 6.61 & 2.9 & 1.8 & N.A. & 0.0 & 0.0 & 0.0 & 0.0 & 0.0 & 0.0 & 0.0 \\
\hline 9 & 19.58 & 4.0 & 3.1 & $3,10,11$ & 2.0 & 35.5 & 36.6 & 27.2 & 4.4 & 2.2 & 2.5 \\
\hline 10 & 27.28 & 3.8 & 4.6 & 3,11 & 1.2 & 5.5 & 17.8 & 17.8 & 0.6 & 1.0 & 1.8 \\
\hline 11 & 35.34 & 4.3 & 5.1 & $4,5,6$ & 1.9 & 88.1 & 41.2 & 33.2 & 9.6 & 2.1 & 3.5 \\
\hline $12^{*}$ & 16.34 & 3.9 & 3.7 & 3,4 & 1.7 & 48.5 & 24.6 & 35.9 & 4.8 & 1.4 & 3.5 \\
\hline 13 & 60.53 & 4.1 & 6.3 & $\begin{array}{c}3,6,7,8 \\
9,10,11\end{array}$ & 13.6 & 104.7 & 233.6 & 48.5 & 11.1 & 21.8 & 3.6 \\
\hline $14^{*}$ & 28.30 & 3.8 & 7.6 & $4,5,6$ & 3.3 & 5.1 & 41.3 & 15.7 & 0.7 & 2.4 & 1.4 \\
\hline $15^{\star}$ & 22.26 & 3.2 & 4.1 & $4,5,9$ & 2.4 & 7.3 & 24.4 & 1.2 & 0.7 & 1.6 & 0.1 \\
\hline
\end{tabular}

demonstrated that the needle selection pattern offers comparable dose to HR-CTV with consistent reduction in dose to OARs. The median dose to the rectum, bladder, and sigmoid decreased by $0.20-0.60$ Gy per fraction. A standard loading procedure for the first fraction in patients with small HR-CTVs should include needles in lateral channels $4,5,9$, and/or 10 inserted $3 \mathrm{~cm}$ deep, with dwell times $<20 \%$ of the combined tandem and ring dwells. The exact percentage of dwell times in the interstitial needles is patient-dependent, and should be determined during planning. It is expected that adjustments would be made for differing shapes and clinical scenario of HR-CTV at the time of brachytherapy. Training is of critical importance in execution of the highest quality cervical brachytherapy boost, and currently there are few data to offer practical initial needle loading [14]. These results should provide insights into optimal needle insertion and dwell times for hybrid applicator, and may help decrease limitations in adopting hybrid IC/IS into more clinical practices.

Dosimetric improvements of the combined IC/IS plans were expected, and such benefits are seen in previous studies of hybrid applicators $[6,7,15]$. The focus of this study was to maintain $\mathrm{D}_{90 \%}$ to maximize the benefit to OAR doses, and to produce benefits in OAR doses on a larger scale than this shown in previous studies. The number of needles suggested for a standard loading procedure was similar, if not less than what is suggest- ed in a previous study on Vienna hybrid applicator [6]. The channel numbers suggested for needle insertion, i.e., $4,5,9$, and/or 10, in a standard loading procedure for Vienna-style applicator, are similar in anatomical locations to channel numbers in other hybrid applicators, such as Utrecht-style IC/IS tandem-ovoid applicator, where only lateral channels are available [7]. In summary, many results from this study either agree with, or improve upon findings from previous dosimetric studies.

Our results are comparable to clinical series of implementation of ring IC/IS applicators. The EMBRACE investigators compared intracavitary vs. hybrid IC/IS applicators (both Vienna-style and Utrecht-style), and found that hybrid ring IC/IS applicators provided the best therapeutic ratio. IC/IS applicators led to an increase of HRCTV $\mathrm{D}_{90 \%}$, comparable bladder $\mathrm{D}_{2 \mathrm{cc}}$ and sigmoid $\mathrm{D}_{2 \mathrm{cc}}$ and 2 Gy decrease of cumulative rectum $\mathrm{D}_{2 c c}$ dose, using an average of 4.8 needles [16]. In our study, by maintaining a consistent $D_{90 \%}$ of at least $85 \mathrm{~Gy}$, we demonstrated that the rectal $\mathrm{D}_{2 c c}$ can be reduced by approximately $0.5 \mathrm{~Gy}$ per fraction, corresponding to a cumulative 2-2.5 Gy decrease of the $\mathrm{D}_{2 c \mathrm{c}}$ to the rectum. Walter et al. used a hybrid IC/IS split-ring commercially-available Venezia applicator (Elekta AB; Stockholm, Sweden), and found that adding needles decreased rectal dose nearly by $4 \mathrm{~Gy}$ and increased HR-CTV dose [17]. Other series demonstrate consistent improvement in the therapeutic ratio and re- 
port on general number of needles used; however, the studies do not offer initial potential needle positions or insertion depths.

Crossley et al. performed a virtual re-planning study using Vienna-style applicator, also demonstrating improved sparing in all OARs studied, with comparable HR-CTV coverage [9]. There were a median number of 2 needles used in each re-plan, with a maximum $4 \mathrm{~cm}$ needle insertion depth. Our study builds on this previous report significantly by offering granular information regarding optimal initial needle selection (lateral needle positions preferred), expected percentage of weighting of loading, validation of digital re-planning approach using a second set of clinically treated IC/IS patients in our clinic, and suggested depth of insertion for our average-sized HR-CTV (generally, non-bulky HR-CTVs). In our current study, in contrast, all patients were planned with MRI for each fraction, and there was an intent to keep HR-CTV coverage comparable.

There are potential limitations of our study. The main limitation of this study is the use of a template of straight needles when re-planning (Figure 2). The deviation of straight needles from clinical IC/IS plans was shown to be small, and similar in size to other setup and planning uncertainties. However, needle deviations may vary in clinics with different insertion techniques, applicators, immobilization, and use of pre-planning. The sample size of this study was small, but the results are impactful enough to suggest a standard initial loading procedure for planning with a ring hybrid. The results of the study suggest a standard loading procedure of needles 4, 5, and 9. However, likely due to sample bias (for example, asymmetric HR-CTVs), it was presumed that needle position 10 would be similarly useful for initial standard loading. We noted there was an asymmetry in our HR-CTVs, as reflected by the increase in the left point A doses and decrease in the right point A doses with IC/IS re-plans (Table 1). The median HR-CTV volume in this study was $19.6 \mathrm{cc}$, suggesting that initial lateral standard needle insertion largely applies to patients with non-bulky HR-CTVs. Finally, it should be emphasized that there are variations in planning between institutions and between planners. Many of the studied plans could have met OARs tolerances using fewer or different needles, but the goal of the present study was to demonstrate the achievable dosimetric benefit of IC/IS and to offer practical needles selection; therefore, any needles that provided dosimetric benefit were applied.

\section{Conclusions}

The results of this study support previous publications by offering practical recommendations on needle placement, demonstrating dosimetric improvement to OARs using a ring IC/IS applicator compared to a ring IC-only applicator. Lateral needle positions inserted approximately $3 \mathrm{~cm}$ are suggested to be optimally used for initial needle selections in non-bulky HR-CTVs; although, caution must be exercised due to proximity of interstitial needles to sensitive patient's anatomy. We demonstrate that parallel ring IC/IS needles are subject to minimal deviation. Training is of immense importance in utilizing new applicators, and all efforts should be taken to reduce barriers in performing the highest quality cervical brachytherapy. We are hopeful that as IC/IS applicators become more common in clinics and that dosimetric improvement and standard loading procedures will be better documented.

\section{Disclosure}

NKT reports advisory board fee from the Varian Medical Systems and consulting from the Boston Scientific. Institutional review board approval was required and granted.

\section{References}

1. Chino J, Annunziata CM, Beriwal S et al. Radiation therapy for cervical cancer: executive summary of an ASTRO clinical practice guideline. Pract Radiat Oncol 2020; 10: 220-234.

2. Tanderup K, Fokdal LU, Sturdza A et al. Effect of tumor dose, volume and overall treatment time on local control after radiochemotherapy including MRI guided brachytherapy of locally advanced cervical cancer. Radiother Oncol 2016; 120: 441-446.

3. Palhares DMF, Marconi DM, Azevedo TLD et al. Predicting the necessity of adding catheters to intracavitary brachytherapy for women undergoing definitive chemoradiation for locally advanced cervical cancer. Brachytherapy 2018; 17: 935-943.

4. Viswanathan AN, Thomadsen B. American Brachytherapy Society consensus guidelines for locally advanced carcinoma of the cervix. part i: general principles. Brachytherapy 2012; 11: 33-46.

5. Kirisits C, Lang S, Dimopoulos J et al. The Vienna applicator for combined intracavitary and interstitial brachytherapy of cervical cancer: design, application, treatment planning, and dosimetric results. Int J Radiat Oncol Biol Phys 2006; 65: 624-630.

6. Fokdal L, Tanderup K, Hokland SB et al. Clinical feasibility of combined intracavitary/interstitial brachytherapy in locally advanced cervical cancer employing MRI with a tandem/ring applicator in situ and virtual preplanning of the interstitial component. Radiother Oncol 2013; 107: 63-68.

7. Nomden $\mathrm{CN}$, de Leeuw AAC, Moerland MA et al. Clinical use of the utrecht applicator for combined intracavitary/ interstitial brachytherapy treatment in locally advanced cervical cancer. Int J Radiat Oncol Biol Phys 2012; 82: 1424-1430.

8. Fokadal L, Sturdza A, Mazeron R et al. Image guided adaptive brachytherapy with combined intracavitary and interstitial technique improves the therapeutic ratio in locally advanced cervical cancer: analysis from the retroEMBRACE study. Radiother Oncol 2016; 120: 434-440.

9. Crossley N, Tipton C, Meier T et al. The value of hybrid interstitial tandem and ring applicators for organ at risk dose reduction in small volume cervical cancer. J Contemp Brachytherapy 2020; 12: 12-16.

10. Harkenrider MM, Surucu M, Harmon G et al. Early outcomes of a hybrid IC/IS applicator for a new MRI-based cervical brachytherapy program. Brachytherapy 2018; 17: 187-193.

11. Malajovich I, Anamalayil S, Dolney OV et al. Techniques for and uncertainties of MRI-based reconstruction of titanium tandem and ring brachytherapy applicators. Brachytherapy 2020; 19: 651-658.

12. Viswanathan AN, Beriwal S, De Los Santos JF et al. American Brachytherapy Society consensus guidelines for locally advanced carcinoma of the cervix. part ii: high-dose-rate brachytherapy. Brachytherapy 2012; 11: 47-52. 
13. Petric P, Hudej R, Music M. MRI assisted cervix cancer brachytherapy pre-planning, based on the insertion of the applicator in para-cervical anaethesia: preliminary results of a prospective study. J Contemp Brachytherapy 2009; 1: 163-169.

14. Tan LT, Tanderup K, Kirisits C et al. Education and training for image-guided adaptive brachytherapy for cervix cancer - The (GEC)-ESTRO/EMBRACE perspective. Brachytherapy 2020; 19: 827-836.

15. Schnell E, Thompson S, Ahmad S et al. Dosimetric considerations when utilizing Venezia, Capri, Rotte double tandem, and tandem and ring with interstitial needles for the treatment of gynecological cancers with high dose rate brachytherapy. Med Dosim 2020; 45: 21-27.

16. Serban M, Kirisits C, de Leeuw A et al. Ring versus ovoids and intracavitary versus intracavtary-interstitial applicators in cervical cancer brachytherapy: results from the EMBRACE I study. Int J Radiat Oncol Biol Phys 2020; 106: 1052-1062.

17. Walter F, Maihöfer C, Schüttrumpf L et al. Combined intracavitary and interstitial brachytherapy of cervical cancer using the novel hybrid applicator Venezia: clinical feasibility and initial results. Brachytherapy 2018; 17: 775-781. 